\title{
Quaderni
}

QUADERNI Communication, technologies, pouvoir

76 | Automne 2011

Les promesses de la biodiversité

\section{La Trame verte et bleue et son public}

Florian Charvolin, Raphaël Mathevet et Ruppert Vimal

\section{(2) OpenEdition}

\section{Journals}

Édition électronique

URL : http://journals.openedition.org/quaderni/141

DOI : 10.4000/quaderni.141

ISSN : 2105-2956

Éditeur

Les éditions de la Maison des sciences de l'Homme

Édition imprimée

Date de publication : 1 septembre 2011

Pagination : 67-78

Référence électronique

Florian Charvolin, Raphaël Mathevet et Ruppert Vimal, « La Trame verte et bleue et son public », Quaderni [En ligne], 76 | Automne 2011, mis en ligne le 01 septembre 2014, consulté le 30 avril 2019. URL : http://journals.openedition.org/quaderni/141 ; DOI : 10.4000/quaderni.141 


\section{$D$ ossier}

\section{la Trame verte et bleue et son public}

\section{Florian Charvolin}

UMR 5283 Centre Max Weber, CNRS, Saint-Étienne

\section{Raphaël Mathevet}

UMR 5175 Centre d'Écologie Fonctionnelle et Évolutive (CEFE), CNRS, Montpellier

\section{Ruppert Vimal}

UMR 5175 Centre d'Écologie Fonctionnelle et Évolutive (CEFE), CNRS, Montpellier
En 2007, le Grenelle de l'Environnement s'imposait comme un vaste chantier participatif visant à étoffer la politique environnementale française. Parmi les divers groupes de travail mis en place, le groupe «Biodiversité » proposait alors de « construire la Trame verte nationale, tissu vivant du territoire, qui assure les continuités et les proximités entre milieux naturels et permet aux espèces de circuler et d'interagir et aux écosystèmes de fonctionner $》$ (MEEDDM, 2007).

Très rapidement devenue un enjeu majeur de la politique grenellienne, l'avènement de la Trame verte et bleue (TVB) en France n'est pas sans lien avec celui du concept de réseau écologique développé depuis les années 1980 dans de nombreux pays européens notamment (Jongman et Kristiansen 2001).

Reconnaissant le besoin de protéger l'ensemble des milieux nécessaires au cycle de vie des espèces, ce concept suppose la mise en œuvre de stratégies de conservation de la biodiversité qui dépassent largement les limites des dispositifs d'aires protégées pour s'étendre sur l'ensemble du territoire. Ainsi le concept de réseau écologique est une opportunité pour créer du lien entre conservation de la biodiversité et aménagement du territoire (Opdam et al. 2006) et finalement donner du sens à la notion de développement durable. Dans la pratique, la planification des réseaux se base largement sur le modèle « tâches, corridors, matrice » issu de travaux d'écologie du paysage de Forman et Godron (1986) et suppose l'identification de trois composantes : les « cœurs de biodiversité », leur « zone tampon» et les « corridors » qui permettent de les relier (Jongman et Pungetti, 2004). 
Nous proposons d'étudier dans cet article la déclinaison de ce modèle dans le travail d'expertise politico-scientifique de mise en place de la TVB, dans deux dispositifs locaux ${ }^{1}$. Il s'agit, d'une part, de l'initiative du Parc naturel régional (PNR) de la Narbonnaise en Méditerranée visant à l'élaboration d'une méthode reproductible d'identification et de délimitation de la TVB ainsi que la définition d'un plan d'action, et d'autre part, du projet porté par les cinq PNR de la Région PACA dans le cadre d'un appel d'offre du ministère et dont l'objectif était la définition d'une méthode de gouvernance de la biodiversité et des conditions d'acceptabilité et de faisabilité d'une TVB au niveau régional.

Dès avant la concrétisation sur le terrain de périmètres et de corridors, les deux initiatives dessinent un public auquel elles destinent la trame, un public putatif, comme incorporé en pensée et dans l'imaginaire des experts, des élus, des scientifiques qui composent les principaux acteurs des deux initiatives (Ellis et al., 2009). C'est de ce public dont il sera question dans ce qui suit. Ce public anticipé et imaginé est enchâssé dans la technicité des débats. L'avantage de la comparaison entre les deux dispositifs est alors de voir varier les termes sur lesquels portent les débats et les publics anticipés en conséquence.

Dans ce travail, nous avancerons la thèse que les deux dispositifs se distinguent selon le schéma suivant : l'un, composé d'un groupe de 100 acteurs clés concernés de par leurs domaines d'action par la mise en place d'une TVB dans la région, est plutôt un forum de discussion multiagents ; l'autre, regroupant principalement des experts de la biodiversité, un comité technique de visualisation de la biodiversité adossé sur des cartes de continuité écologique. Il s'agit de voir comment ces deux dispositifs fabriquent du public renvoyant les débats, d'une part, aux modalités de construction et à la rhétorique de l'inscription scientifique comme a pu l'exposer Bruno Latour; et d'autre part à un forum de points de vue et de débats sur les valeurs comme a pu l'analyser Jürgen Habermas.

Nous serons particulièrement sensibles à la qualité des considérations sur laquelle s'appuient les « points de vue » des uns et des autres, certains alimentant des imaginaires plus ou moins prospectifs et les autres des « vues de l'esprit» scientifiques. Mais dans les deux cas, notre observation a porté sur les débats et sur les documents y afférant et notre définition du « public » est l'extrapolation des controverses permettant à une TVB de progressivement se dessiner dans les débats.

\section{L'espace et la liste : la rencontre entre scien- tifiques et politiques}

Les écologues ou les naturalistes ont souvent été associés à des initiatives de réglementation des espaces sur le territoire national (Charvolin, 2003). Il fut un temps où les naturalistes travaillant sur les écosystèmes s'accommodaient bien d'une injonction publique à définir des sanctuaires de nature protégés de la population comme des zones géographiques à l'écart, même si de nombreux travaux nuancent aujourd'hui cette injonction (Mauz, 2002). L'origine de la biodiversité a été décrite en remontant aux notions de réseaux écologiques (Jongman et Pungetti, 2004), à l'attrait pour une écologie des îles (MacArthur et Willson, 1967) avant cela, et en en restituant l'histoire usuelle qui l'a fait remonter à Wilson 
(Takacs, 1996. La biodiversité est associée à des politiques publiques de zonage de plus en plus élaborées, notamment après la directive européenne Habitat-Faune-Flore de 1992, et la TVB s'inscrit dans cette collaboration entre instances scientifiques et programmation politique des territoires.

L'approche en termes de corridor, de réseau écologique ou de TVB, est cependant un peu différente d'une approche par zonage, il s'agit de trouver ce que l'on appelle la « continuité écologique » dans les travaux officiels du Grenelle. Rien de moins immédiat que cette continuité dont on se préoccupe lorsqu'elle menace d'être perdue et qu'il faut par conséquent assurer par des relations à plus ou moins grande distance du public. La continuité écologique sert de base à la généralisation de l'enjeu de la biodiversité, pour l'urbain comme le rural, comme un concernement, qui pour ne pas être direct, est de proximité. Le caractère public de la biodiversité devient alors patent. Toutefois il faut comprendre combien l'agencement des informations sur la TVB est en rupture avec l'expérience attachée au «public » tel qu'on le définit d'habitude comme personne du commun ; il le suppose certes, mais au niveau de l'imaginaire qui reste largement à l'œuvre dans cette négociation encore virtuelle de la future TVB.

Du même coup s'affrontent deux logiques d'intégration du public. D'une part, l'une le pose comme un destinataire final d'un processus de quantification qui exclut le profane de la pratique en train de se faire. L'élément de rupture avec le public, par exemple l'amateur ou le profane, est consommé dans la notion de « donnée » quanti- fiable, qui est appelée par la notion de « réservoir de biodiversité » qui cumule une dimension spatiale, un contenant, et une dimension quantitative. Or, «l'histoire de notre savoir sur la biodiversité est tout d'abord une histoire de collecte et de collection» (Kohler, 2006). Dire « réservoir de biodiversité » c'est dire « donnée quantifiable ». Mais d'autre part, l'autre logique retrouve le public en pratique dans le cercle des parties prenantes, dès lors que la TVB devient un enjeu de négociation pluri-acteurs dans lequel sont conviés au même titre, les spécialistes, les politiques ou les associations. Il y a donc un jeu sur le public imaginé selon que l'on confine la pratique de montage de la TVB à la production controversée de la « donnée », ou que l'on ouvre ce montage à une scène d'argumentation contradictoire ouverte à la diversité des points de vue et des sources de légitimité.

L'établissement des deux dispositifs étudiés pour cet article se fonde donc sur une « bio-diversité » assimilée à une « data diversity » pour reprendre les termes de Bowker (2000), à travers laquelle on espère représenter fidèlement la nature et l'évolution vertueuse qu'on espère d'elle. Il existe de nombreux débats sur les fondements et les possibilités de «quantifier la distribution spatiale des enjeux » de la protection des lieux (Vimal et al., 2011). Bref, la biodiversité, y compris dans les dispositifs régionaux de son traitement, est affaire de quantification, de dénombrement, de collection, et suppose que la standardisation des données ait déjà fait son office pour rendre capable une plateforme politico-scientifique d'opérer des choix et de construire des corridors écologiques notamment, sans en référer au moment de la collecte. 


\section{Forum et assemblée cartographique}

Les deux cas étudiés que l'on va présenter maintenant sont dans cette phase de brassage de données.Ils permettent de faire ressortir des cartes ou des principes de travail ou encore des logiques d'intervention sur le territoire. On le répète, cette phase est conditionnée par le caractère standard, autonomisé et combinable de la donnée naturaliste ou écologique disponible pour le territoire. Elle entretient donc une distance manifeste avec la participation du public et pourtant elle induit un public putatif à la TVB. Il s'agit d'identifier des corridors écologiques en particulier. Il est question de parachever l'œuvre de Natura 2000 et des Znieff (Zone Naturelle d'Intérêt Ecologique, Faunistique et Floristique) pour finir de mailler le territoire.

On va voir cependant que le maillage en question relance pour certains la controverse sur le mode d'agrégation des données, et sur le conflit latent entre principe de spatialisation et les modalités de collecte de données sur le terrain. C'est cette controverse qui anime le collectif que nous avons appelé « assemblée cartographique ». D'autres acteurs ont délégué à une cellule technique le soin d'assurer la compatibilité de données, et passent du temps sur les principes permettant au collectif de se maintenir. Nous avons appelé ce collectif un forum.

Dans le cas de l'assemblée cartographique, celui du parc naturel régional de la Narbonnaise, les explorations scientifiques hésitantes seront au premier plan, à travers la question centrale de la mise au point de cartes. Dans le cas du forum, qui correspond assez bien au dispositif développé en région PACA, il est question de ce qu'il est raisonnable d'exiger en tant que scientifique dans une mission d'expertise, d'un point de vue qui n'entre pas dans la controverse savante.

\section{L'assemblée cartographique du PNR de la Narbonnaise}

Le dispositif du PNR de la Narbonnaise se distingue par le fait qu'il est majoritairement composé d'experts en lien direct avec la conservation de la nature. Poursuivant l'objectif explicite de délimiter la TVB du territoire du Parc, une part de l'expertise technique est externalisée : un bureau d'étude (BE) est mandaté pour produire des cartes à destination des naturalistes et des scientifiques censés les amender au vu de leur domaine de compétence respectif.

Ici le collectif s'inspire des recommandations nationales émanant du Comité opérationnel (Comop) TVB ainsi que des diverses expériences en cours en Europe. L'objectif est de visualiser, à l'aide de cartes, les éléments constitutifs d'un réseau écologique : les corridors et les cours de nature qui délimitent les continuités écologiques. C'est ainsi que le suivi des réunions donne à voir comment la représentation cartographique, la spatialisation des continuités écologiques manifeste les compétences spécialisées développées par les acteurs limitant ainsi le débat aux controverses techniques et scientifiques liées au contenu même de la TVB. La discussion suivante ${ }^{2}$, autour d'un premier regroupement des classes d'occupation du sol proposé par le bureau d'étude, permettra d'illustrer notre propos :

«Centre National de la Recherche Scientifique 
(CNRS) : Où sont classées les falaises?

- Bureau d'Étude (BE) : Elles sont dans la classe "pelouses ». De toute façon, en termes de surface, elles ne sont pas forcément intéressantes.

- Ligue pour la protection des oiseaux (LPO) : Oui mais ça peut être des barrières, des obstacles pour certaines espèces.

- BE : Oui mais on risque de ne plus les voir si on les isole dans une classe. À ce stade, l'objectif est de définir les grandes zones, de voir la relation entre ces zones et d'analyser le contraste.

- Office National de la Chasse et de la Faune Sauvage (ONCFS) : Ça suppose d'avoir une échelle plus fine après, parce qu'au sein des forêts par exemple y a plein de choses différentes notamment en termes de structure.

- Office pour les Insectes et leur Environnement (OPIE) : Pour l'approche espèces, vous allez aussi utiliser cette échelle? Moi ça me gène qu'il $n$ 'y ait pas les falaises.

- BE : Le but ici est de réfléchir sur les grandes zones et la connexion qu'il y a entre ces zones. "

Ici la représentation cartographique se confronte aux réalités de terrain exprimées par les divers acteurs en présence. Si les participants semblent s'accorder sur le fait que l'appréhension $\mathrm{du}$ fonctionnement des systèmes écologiques à un niveau si global pose problème, on devine cependant que ce consensus apparent naît des spécialités de chacun : les falaises dont la morphologie ne permettrait pas la visualisation sur une carte sont pourtant, aux yeux des ornithologues, un élément central en termes de ruptures de continuités pour l'avifaune. L'expert de l'ONCFS se fait le représentant de la diversité des structures en milieu forestier et l'entomologiste (OPIE) semble déjà prévenir de l'inadé- quation de cette approche pour les invertébrés.

C'est ainsi qu'au sein de ce dispositif, la polarisation de l'expertise, consistant à placer d'un côté le bureau d'étude pour produire les cartes et de l'autre les experts pour en faire la critique, a ancré le collectif dans une démarche très technique limitant le partage des enjeux et d'autres dimensions de la TVB. Le résultat qui émerge de cette première confrontation du modèle TVB aux réalités du territoire est peu innovant, contesté et jugé sans grand intérêt par certains des acteurs (Vimal et Mathevet, 2011).

Dans ce premier dispositif, nous retiendrons que pour les scientifiques et experts il s'agit de faire en sorte d'assurer une représentation fidèle des objets dont ils sont spécialistes. On entre dans les contenus, chacun doit y retrouver ses petits mais alors on hésite sur les représentations cartographiques à donner et au final, du moins au terme de cette première étape du projet, la controverse reste ouverte, pleine et entière. Le public putatif de cette controverse apparaît comme facteur humain impactant le territoire à cartographier et suscite le scrupule des scientifiques pour stabiliser entre eux, une représentation de la TVB.

\section{Le forum de PACA}

Dans le forum PACA, l'enjeu central de la « participation » a contraint les pilotes de la démarche à positionner le groupe général composé des socioprofessionnels concernés par la mise en place de la TVB dans la région, comme seul légitime pour définir le contenu de la TVB et les moyens d'action. Les experts mandatés en tant que tels sont ainsi restés en situation 
d'accompagnement et garants de la place non hégémonique de l'expertise technique comme en témoigne cette intervention d'un représentant de l'Agence Régionale Pour l'Environnement : « On a trop d'incertitudes techniques. On est au stade recherche et développement. Il faut que les gens qui présentent ce résultat soient capables d'expliquer cette démarche et cette incertitude ». C'est ainsi que l'absence d'une vision préconçue de la TVB et de la manière de la prendre en compte a favorisé un débat au sein du groupe général qui s'est rapidement détaché à la fois d'une approche naturaliste et d'une approche spatiale de la TVB.

Le projet PACA s'inscrit dans une vision plus globale des enjeux de conservation qui met l'accent sur « la nécessaire prise en compte de la biodiversité dans les politiques publiques et notamment dans l'aménagement du territoire ». Dans la définition proposée par le groupe général, il est d'ailleurs frappant de voir que, si la TVB semble parfois faire écho à une réalité biologique plus qu'à un outil stratégique, rien ne permet cependant de définir ce qui la compose au-delà de la phrase suivante : « Son approche intègre l'ensemble des zones naturelles, des zones agricoles et des zones urbaines $»$. Ainsi le collectif n'a pas vraiment cherché à se doter d'une représentation spatiale de ce qu'est la TVB en termes de réalité écologique. Sur la base du porter à connaissance ayant permis d'appréhender la complexité de la nature et des interdépendances qui la caractérisent, le groupe reconnait implicitement l'existence d'un réseau écologique et, dès lors, l'enjeu et le sens du projet relèvent plus de l'organisation humaine et de l'ingénierie sociale nécessaire à sa préservation que de sa stricte caractérisation.
Dans ce forum, pour appréhender le rôle de l'expertise, il faut être en mesure d'analyser ce qui relève de l'expertise technique, d'un savoir faire, et ce qui relève d'une expertise scientifique au sens des connaissances, des savoirs. En effet, si nous avons vu que la mise en retrait de la technique au profit de la participation a permis de faire émerger le caractère multidimensionnel du concept de TVB, c'est aussi parce qu'en positionnant la technique en accompagnement, le débat cognitif a pu avoir lieu. Alors que dans l'assemblée cartographique du PNR, le modèle jouait le rôle d'une connaissance à stabiliser, ici, les scientifiques universitaires présents dans les différentes instances ont assuré les porter à connaissance définissant les grands enjeux écologiques - ce qui menace de ne plus existeret dépassant ainsi la simplification qu'une discussion technique n'aurait pas manqué de privilégier.

Dans ce dispositif, nous retiendrons que les scientifiques académiques ou issus des diverses institutions en charge de la biodiversité régionale sont des acteurs parmi d'autres. On n'entre pas vraiment dans les contenus factuels de la TVB. On se met d'accord sur les garanties que les scientifiques, comme autorité légitime, sont en position d'exiger pour que le débat ait une teneur qui corresponde à leur attente ou intérêt, par rapport à l'intérêt d'autres participants ; et du coup on assiste à une sorte de démocratie participative, où l'on gère finalement les hommes plutôt que les contenus.

On voit ici qu'avec la TVB, on n'est pas nécessairement dans du « tout participatif » ou du tout « expertise technique » mais que les formes de l'expérimentation socio-technique diffèrent en 
fonction des lieux pour le même instrument d'action publique. À ce stade nous pouvons résumer le propos en disant que dans un cas l'assemblée cartographique se sent des « obligations » par rapport à ce qu'elle pose comme ce qui l'oblige, à savoir une représentation précise de la nature. Dans l'autre cas les scientifiques interviennent plutôt en représentation, comme défendant leur institution, et déclinant des « exigences », au même titre que les politiques, les administratifs etc. Et il n'est pas étonnant que la première des scènes se caractérise par son hésitation, tandis que l'autre est plus assurée et décisionnelle (Stengers, 2006).

\section{Quel public pour la TVB?}

Dans nos deux exemples, on observe que le public était à la fois plus ou moins extériorisé aux scènes régionales de mise en place de la TVB et pourtant qu'une « conception » du public, une certaine place du public était supputée pour soutenir le travail d'articulation entre science et politique dans le cas du PNR de la Narbonnaise ou de PACA. À présent, nous allons voir combien cette place varie mais également combien est limitative notre conception substantielle du public, comme une arène, une audience, un groupe de personnes partageant des qualités différentes de l'administration, du scientifique, du politique etc. En tant que trame donnant tout son sens normatif à la biodiversité sur le terrain, la TVB est, en l'état de ce que nous avons pu observer, un vœu pieux. Elle est encore dans les cartons, ce qui n'empêche pas qu'elle ait une consistance programmatique et c'est à ce stade que les considérations sur le public de la TVB peuvent intervenir le plus aisément, au moment de sa virtualité et avant qu'elle se fasse sentir concrètement. Mais alors elles rentrent en résonance avec le projet scientifique lui-même d'inventorier toute la nature dans sa diversité.

\section{L'interconséquentialité en question}

Avec la TVB, les espaces dédiés à la conservation intègrent ainsi à nouveau l'espace des sociétés : leurs territoires. Avec la notion de réseau écologique ou de corridor écologique la nature ainsi " déconfinée » devient une « socio-nature », un système « socio-écologique » (Walker et al. 2004). Elle conduit à restaurer dans les choses de la nature une des raisons de notre solidarité, de notre saisie en tant que public, mais un public pragmatique et non pas substantiel. Nous allons nous en expliquer maintenant.

Le public peut être conçu comme un processus qui advient dans certaines circonstances quand le jeu de la distance augmente entre les gens ou leur milieu et où la vigilance doit alors redoubler. D'un point de vue écologique, le corridor dont la TVB fait une de ses bases, installe également un réseau de liens entre des espaces de plus en plus fragmentés et tire sa pertinence de cet impact d'une tâche d'habitat sur une autre, quelquefois à relier sur des distances importantes. Or cette interrelation, dont le génie écologique cherche à faire l'analyse, et dont le conservationisme cherche à gérer au mieux l'évolution (MEA, 2005), est significative d'une qualité que John Dewey a qualifiée de «publique ». Pour comprendre ce caractère public, on peut reprendre la définition pragmatique du problème public sous la forme deweysienne des conséquences indirectes des actions des uns sur les autres. Ici 
cette interconséquentialité se conçoit uniquement selon la condition qu'elle soit indirecte et donc qu'il faille des médiations pour la saisir voire la gérer. Or l'approche TVB énonce une solidarité écologique, une interdépendance à distance plus ou moins grande des éléments naturels comme les espèces ou les écosystèmes (Mathevet et al., 2010). La prise en compte de la nature ne peut alors être cantonnée à une localité sans en sortir, comme peuvent l'être certaines opérations de zonage des sanctuaires de nature. Natura 2000 avait déjà montré le chemin, mais la TVB, en tant que trame, radicalise le maillage. Du même coup, ce dont les écosystèmes constituent l'environnement personnel, à savoir les individus, nous et vous, se connectent, et ce sur de plus en plus grandes distances. Nous voici tous concernés mais de manière plus ou moins indirecte, ce qui établit très exactement un « public » au sens deweysien.

Cette définition processuelle et pragmatique du public va, on l'aura compris, à l'encontre d'une définition substantielle du public comme « l'habitant », «l'homme de la rue » ou encore le « profane ». De ce fait, on peut retrouver du public aussi bien dans une assemblée cartographique d'experts, et dans un forum plus ouvert de porteurs d'enjeux. En l'occurrence, sous le terme de public, se déclinent, dans ces deux cas, des dimensions très différentes mais finalement comparables sous l'angle politique. À l'image de Boyle (Shappin et Schaffer, 1993) qui créait un discours politique tout en évacuant le politique et de Hobbes qui reformulait une politique scientifique tout en évacuant la science expérimentale (Latour, 1991), l'exemple de la TVB dans le PNR de la Narbonnaise montre comment en évacuant le politique pour en faire une étape future, on ne parvient pas à mettre d'accord les parties prenantes - en « boite noirisant » la carte ; tandis que l'exemple de la TVB en PACA montre une requalification des sciences de la conservation dans la politique.

\section{De la pompe à air à la carte aux quatre vents}

L'exemple du PNR de la Narbonnaise donne à voir comment la carte destinée à représenter la TVB est jointe aux modalités de production du savoir à travers ses conditions d'inscription. Elle est du travail incorporé à même le visuel et les éléments graphiques produits (Latour 1985). Troublée par une quête de l'opérationnel, l'assemblée cartographique s'est focalisée sur la dimension technique des enjeux. Elle a négligé la clarification de ses objectifs et a sous-évalué l'importance d'une réflexion sur ce nouveau concept de TVB (Vimal et Mathevet, 2011). L'élaboration de la carte peut être analysée comme une inscription; elle s'inscrira dès lors dans un réseau socio-technique structuré par des relations d'intéressement pour le contenu cognitif de l'inscription mais également d'intérêt politique pour le standard ainsi produit etc. (Callon, 1986). Le jeu relationnel qui s'est établi entre le mandataire, l'opérateur technique, les scientifiques et experts montre comment l'élaboration de la TVB a été constamment confrontée à des problèmes de construction et de négociations, d'imposition hiérarchique de points de vue. La cartographie de la TVB n'a alors eu de cesse de tituber entre le projet d'intelligibilité et de visibilité de la connaissance et les lacunes de la rationalité scientifique et technique censée la concrétiser. Cela se traduit par une carte finale controversée quant à ses fondements. Ainsi, dans ce contexte, 
la carte est restée davantage un objet-média, c'està-dire un objet qui concourt à la transmission de connaissances et de recommandations plutôt qu'un objet-médiateur (Vinck, 1999), c'est-àdire un objet qui participe à l'exploration et la transformation de la problématique.

\section{Le Léviathan en mode contextualisé}

Dans l'exemple de la TVB en région PACA, partant d'une remise en cause fondamentale d'une simple application du modèle « taches, corridors, matrice $»^{3}$, le comité de pilotage a créé un forum pour que la TVB soit un objet intermédiaire entre les parties prenantes pour discuter et partager les connaissances relatives à la dynamique de la biodiversité et réfléchir à la complexité politique et technique des décisions. Ce choix souligne un fléchissement conséquent de l'objet TVB, du statut d'objectif vers le réseau écologique à celui de moyen pour établir une gouvernance et réfléchir collectivement à la place de la biodiversité dans l'aménagement du territoire. Le Forum Paca montre ainsi que dans la mesure où les projets de conservation se fondent sur les approches participatives dans une quête de pertinence et d'acceptabilité sociale (Pretty, 1995), l'enjeu est de questionner les conditions de production de consensus (Stengers, 2009).

Ici, les scientifiques et experts techniques n'ont pas fourni au collectif d'acteurs une recommandation mais un constat, une appréciation sur les points d'accord, de controverses et d'incertitudes inhérents au sujet traité. Ce positionnement de l'expertise en situation d'accompagnement a permis aux acteurs de réfléchir collectivement aux enjeux écologiques et aux outils nécessaires à leur prise en considération. Le dispositif a privilégié ainsi une rationalité communicationnelle plus qu'instrumentale (Habermas, 1987) et de ce fait, l'expertise et son usage ne sont plus un enjeu de pouvoir et un produit de conflits entre une variété d'intérêts. Bien au contraire, le collectif s'appuie d'abord sur l'enseignement synthétisé des sciences pour prendre acte de la complexité du vivant, établir un constat propre au territoire, tirer des conclusions qui font sens pour tous et optimiser ensuite la complémentarité des savoirs en présence pour définir une position stratégique. L'expertise est alors en quelque sorte partagée et assurée par l'ensemble du collectif (Callon et Rip, 1992). Ici l'objet TVB, en tant que symbole d'un renouveau des politiques publiques de conservation de la nature, n'est finalement qu'un concept médiateur, un moyen pour faire partager, hésiter ensemble les individus. Cependant à la fin du processus, tout le monde s'accorde à remarquer que si les leviers d'action publique ont été identifiés grâce au dialogue social, il manque une spatialisation permettant de les opérationnaliser. Dans cet exemple le dispositif a mobilisé des acteurs appartenant essentiellement au corps intermédiaire et à une échelle régionale. La question de l'enrôlement de l'ensemble des habitants et acteurs directs pour définir un intérêt général et aboutir à une réelle prise en compte de la biodiversité demeure un objectif à atteindre.

\section{Conclusion}

Au final, la présentation du public putatif de la TVB dans les cas étudiés ne serait pas complète s'il n'était pas question aussi de la politique de l'inventaire et de la base de données. Ce qui est pris pour acquis par le terme même de bio- 
diversité, c'est la quantification de la nature et la possibilité à terme d'en saisir l'exhaustivité. À l'échelle de la région méditerranéenne par exemple, circulent des listes d'espèces déclinant ce qui est estimé comme une quantité fiable de la diversité biologique. L'hétérogénéité est ainsi postulée comme un élément comptable, et dont on peut potentiellement épuiser le dénombrement, là où Kohler (2006), par exemple, note une autre définition de la diversité comme « variabilité » infinie. La tentative de comptage, la statistique suppose alors une politique de la nature qui permette à tous les éléments de s'additionner. Nos deux exemples nous révèlent comment l'objet public TVB demande de mobiliser des concepts, de comprendre et de signifier des processus afin de les projeter sur les territoires de la biodiversité par le truchement de listes et de cartes contraintes par la maîtrise technologique - Système d'information géographique, gestion de bases de données spatialisées. Or, le paradoxe du politique en démocratie est de ne pouvoir se résoudre dans une métrique, ou une statistique, ce que Rancière (1995) appelle le pouvoir de «police». L'effort pour standardiser toujours plus les données et en assurer l'intercomparaison, se fait de plus en plus dans l'ombre portée du travail minutieux de collecte et toute la richesse d'un terrain plus vaste que l'échelle à laquelle on le réduit, qu'il s'agisse de quadrats pour les écosystèmes ou de listes pour les espèces. Gageons que la TVB retrouvera le contact avec la collecte et la population pour en faire une œuvre plus partielle, et peut-être alors plus « partiale » dans sa dénotation d'un projet politique.

\section{$R \cdot \hat{E} \cdot F \cdot \dot{E} \cdot R \cdot E \cdot N \cdot C \cdot E \cdot S$}

G. BOWKER, « Biodiversity, Datadiversity » in Social Studies of Science, vol. 30, n5, 2000, pp. 643-683.

M. CALLON, «Éléments de sociologie de la traduction » in L'année sociologique, n³6, 1986, pp.169-208.

M. CALLON et A. RIP, « Humains, non humains : morale d'une coexistence » in Theys J. et Kalaora B. (Eds), La Terre outragée. Les experts sont formels, Paris, Editions Autrement, 1992.

F. CHARVOLIN, L'invention de l'environnement en France. Chronique anthropologique d'une institutionnalisation, Paris, La Découverte, 2003. R. ELLIS, C. WATERTON, et B. WYNNE, " Taxonomy, biodiversity and their publics in twenty-first-century DNA Barcoding ", Public Understanding of Science, 2009, 19(4) pp. 497-512.

R.T.T. FORMAN et M. GODRON, Landscape Ecology, New York, NY, USA, John Wiley, 1986. J. HABERMAS, Théorie de l'agir communicationnel, Paris, Fayard, 1987.

R.H.G. JONGMAN, et I. KRISTIANSEN, « National and regional approaches for ecological networks in Europe » in Council of Europe Nature and Environment Series, ${ }^{\circ} 110,2001$, pp. 1-86. R. JONGMAN et G. PUNGETTI, « Ecological networks and greenways: concept, design, implementation " in Cambridge studies in landscape ecology, i-xxi, 2004, pp. 1-345.

R. KOHLER, All creatures, Princeton, Princeton Univ. Press., 2006, p. 6.

R. KOHLER, All creatures, Princeton, Princeton Univ. Press, 2006.

B. LATOUR, Nous n'avons jamais été modernes. 
Essai d'anthropologie symétrique, Paris, La Découverte, 1991.

R.H. MACARTHUR et E.O. WILSON, The theory of island biogeography, Monographs in population biology. Princeton University Press, Princeton, NJ, USA, 1967, p. 203.

R. MATHEVET, et alii, « La solidarité écologique: un nouveau concept pour la gestion intégrée des parcs nationaux et des territoires » in Natures Sciences Sociétés, vol. 18, n 4, 2010, pp. 424-433.

I. MAUZ, «Comment est née la conception française des parcs nationaux ? » in Revue de Géographie Alpine, Tome 90, n 2, 2002, pp. 33-44. MEEDDM. Le Grenelle de l'Environnement. Préserver la biodiversité et les ressources naturelles, Synthèse du groupe 2, Paris, MEEDDM, 2007.

MILLENIUM ECOSYSTEM ASSESSMENT, Ecosystems and human well-being, Synthesis, Chicago, Island Press, 2005, p. 11.

P. OPDAM, E. STEINGRÖVER et S.V. ROOIJ, « Ecological networks: A spatial concept for multi-actor planning of sustainable landscapes » in Landscape and Urban Planning, $\mathrm{n}^{\circ} 75,2006$, pp. 322-332.

J.N. Pretty, « Participatory Learning for Sustainable Agriculture » in World Development, n²3, 1995, pp. 1247-1263.

J. RANCIÈRE, La mésentente, Paris, Galilée, 1995.

S. SHAPIN et S. SCHAFFER, Léviathan et la pompe à air, Paris, La découverte, 1993.

I. STENGERS, La vierge et le neutrino, Paris, Les empêcheurs de penser en rond, 2006.

I. STENGERS, Au temps des catastrophes. Résister à la barbarie qui vient, Paris, La Découverte, 2009.
D. TAKACS, The Idea of Biodiversity: Philosophies of Paradise, Baltimore, The Johns Hopkins University Press, 1996.

R. VIMAL, A.S.L. RODRIGUES, R. MATHEVET et J. THOMPSON, « The sensitivity of gap analysis to conservation targets " in Biodiversity and Conservation, vol. 20, n³, 2011, pp. 531-543.

R. VIMAL et R. MATHEVET, « La carte et le territoire : la trame verte et bleue ou la mise à l'épreuve de l'assemblée cartographique » in Cybergeo, 2011, in press.

D. VINCK, Ingénieurs au quotidien. Ethnographie de l'activité de conception et d'innovation, Presses Universitaires de Grenoble, Grenoble, 1999.

B. WALKER, C. S. HOLLING, S. R. CARPENTER et A. KINZIG, « Resilience, adaptability and transformability in social-ecological systems » in Ecology and Society, vol. 9, n², 2004, 5. [URL] http://www.ecologyandsociety.org/vol9/iss2/art5/ 


\section{$N \cdot O \cdot T \cdot E \cdot S R \cdot E \cdot S \cdot U \cdot M \cdot E$}

1. Sur une démarche similaire voir : Pinton F. et al. $L a$ construction du réseau Natura 2000 en France, Paris, La documentation française, 2006.

2. Deux d'entre nous ont pu assister aux réunions de l'assemblée cartographique de la Narbonnaise et du forum PACA en 2009 et 2010.

3. Du fait qu'il négligerait de nombreux aspects du paysage en donnant une image binaire du territoire composé de zones soit propices soit néfastes voir D. Lindenmayer et alii, «A checklist for ecological management of landscapes for conservation » in Ecology Letters, vol. 11, $\mathrm{n}^{\circ} 1,2008$, pp. 78-91.
L'approche de la Trame Verte et Bleue (TVB) énonce une solidarité écologique, une interrelation à distance des espèces ou des écosystèmes qui participent d'une définition processuelle et pragmatique du public. Le suivi de deux dispositifs de mise en œuvre de la TVB promue par le Grenelle de l'environnement montre comment ces dispositifs, bien que différents, fabriquent un public putatif auquel ils destinent la trame, public anticipé et imaginé par les experts, les élus, les scientifiques. Ils dévoilent comment ce public varie dans les deux cas, sans toutefois résoudre la question de la définition d'un intérêt général.

\begin{abstract}
:
The approach of green infrastructure (Trame Verte et Bleue) acknowledges the ecological solidarity and distant interrelationships between species or ecosystems and as such contributes to a processual and pragmatic definition of the public. Based on two case studies of implementation of the ecological network promoted by the environmental roundtable in France, our paper showed how these two different approaches created a putative public for the Green infrastructure, a public anticipated and imagined by the experts, the elected people, and the scientists. These approaches revealed how the public changes in the two cases, without resolving the question of the definition of the general interest.
\end{abstract}

\section{Remerciements}

Nous remercions pour leur accueil et leur intérêt les participants et les animateurs des deux dispositifs étudiés. Cette étude a été financée par l'Union Européenne (contrat 226852, SCALES) et par la Région Languedoc-Roussillon. 